\title{
DIFFERENCE PROPERTIES FOR CONTINUITY AND RIEMANN INTEGRABIITY ON LOCALLY COMPACT GROUPS $\left({ }^{1}\right)$
}

\author{
BY \\ F. W. CARROLL
}

1. Introduction. Unless otherwise specified, $(G,+)$ will always denote a (not necessarily abelian) locally compact topological group. Measure will denote a Haar measure on $G$; if $G$ is compact, it is assumed that $\mu(G)=1$. All functions considered are complex-valued. If $f$ is a function on an open subset $A$ of $G$, then $\Delta_{h} f$ and $\nabla_{h} f(h \in G)$ denote the functions given by $\Delta_{h} f(x)=f(x+h)$ $-f(x)$ and $\nabla_{h} f(x)=f(h+x)-f(x)$ on $A \cap(A-h)$ and $A \cap(-h+A)$, respectively. A function $\Gamma$ on $G$ is said to be additive if $\Gamma(x+y)=\Gamma(x)+\Gamma(y)$ for all $x$ and $y$ in $G$. A function $f$ on an open subset $A$ of $G$ is said to be Riemann integrable if $f$ is bounded on every compact subset of $A$, and if the set of points of discontinuity of $f$ is of measure 0 .

The classes of continuous functions and Riemann integrable functions were among the many classes $C$ of functions on the real line which, by results of de Bruijn $[2 ; 3]$, are known to possess the following difference property: If $f$ is a function on $R$ such that, for each $h \in R, \Delta_{h} f \in C$, then there exists an additive function $\Gamma$ on $R$ such that $f-\Gamma \in C$. It will be shown that these two classes continue to possess such a property, or a stronger property, the so-called local difference property due to Kemperman [6], when the functions are defined on compact groups, or on open subsets of locally compact abelian groups.

In what follows, many statements concerning the class of continuous functions become valid for Riemann integrable functions after slight modification; these changes will be enclosed in brackets [].

2. The compact case. If the group $G$ is compact [and second countable], the class of continuous [Riemann integrable] functions possesses a difference property like de Bruijn's under conditions weaker than might be expected. $G$ need not be abelian, and it suffices that all right differences (say) are continuous [Riemann integrable], while all left differences are Borel measurable.

THEOREM 2.1. Let $G$ be compact, and let $f$ be a function on $G$ such that, for every $h \in G$,

(i) $\nabla_{h} f$ is Borel measurable, and

(ii) $\Delta_{h} f$ is continuous.

Then there exists an additive function $\Gamma$ on $G$ such that $f-\Gamma$ is continuous on $G$.

Presented to the Society, November 28, 1959; received by the editors December 16, 1960.

(1) This research constitutes a portion of a doctoral dissertation at Purdue University, written under the direction of Professor J. H. B. Kemperman. 
THEOREM 2.2. Let $G$ be compact and second countable, and let $f$ be a function on $G$ such that, for every $h \in G$,

(i) $\nabla_{h} f$ is Borel measurable, and

(ii) $\Delta_{h} f$ is Riemann integrable.

Then there exists an additive function $\Gamma$ on $G$ such that $f-\Gamma$ is Riemann integrable on $G$.

The basic ideas in the proof are to show that the functions $g$ and $\Gamma$ can be defined on $G$ by means of the equations

$$
\begin{aligned}
& g(x)=\int_{G}(f(x)+f(y)-f(x+y)) d \mu(y), \\
& \Gamma(x)=\int_{a}(f(x+y)-f(y)) d \mu(y),
\end{aligned}
$$

so that

$$
f(x)=g(x)+\Gamma(x)
$$

and then to show that $\Gamma$ is additive, while $g$ is continuous [Riemann integrable].

For each $x$, the integrands in (2.1) and (2.2) are measurable functions of $y$, and their sum is $f(x)$. As soon as it is shown that the integrand in (2.1) is a bounded function, both integrands will be bounded, so that both $g(x)$ and $\Gamma(x)$ will be defined. The boundedness of this integrand (jointly in $x$ and $y$ ) follows from taking $\phi_{x}(y)=f(x)+f(y)-f(x+y)$ in Lemma 2.1 below. The formulation of Lemma 2.1, stronger than necessary for the present application, will be used in the next section; the same remark will apply to Lemmas 2.2 and 2.3. The method of proof of this lemma is similar to that of de Bruijn $[3]$.

LemMA 2.1. Let $G$ be compact, and let $\left\{\phi_{\lambda}\right\}$ be a collection of measurable functions on $G$ such that, for each $h \in G$, the values $\left|\Delta_{h} \phi_{\lambda}(y)\right|$ are uniformly bounded in $\lambda$ and $y$. Then $\left|\phi_{\lambda}(y)-\phi_{\lambda}(0)\right|$ is uniformly bounded in $y$ and $\lambda$.

Proof. Let

Since

$$
S_{M}=\left\{h:\left|\phi_{\lambda}(y+h)-\phi_{\lambda}(y)\right| \leqq M \text { for all } y \text { and } \lambda\right\} .
$$

$$
G=\bigcup_{M=1}^{\infty} S_{M}
$$

it follows that $\mu^{*}\left(S_{M}\right)>1 / 2$ for all $M$ sufficiently large, where $\mu^{*}$ denotes the outer measure associated with $\mu$. Let such an $M$ be chosen and kept fixed, let $\lambda$ be fixed, and let

$$
S=\left\{k:\left|\phi_{\lambda}(k)-\phi_{\lambda}(0)\right| \leqq M\right\} .
$$


Clearly $S$ is measurable, and $S_{M} \subset S$, so that $\mu(S)>1 / 2$. For each $y \in G$, $\mu(-S+y)>1 / 2$, so that.

$$
\begin{aligned}
\mu^{*}\left(S_{M} \cap(-S+y)\right) & =\mu^{*}\left(S_{M}\right)+\mu(-S+y)-\mu^{*}\left(S_{M} \cup(-S+y)\right) \\
& >(1 / 2)+(1 / 2)-1=0 .
\end{aligned}
$$

Let $h$ be a point common to $S_{M}$ and $-S+y$. Then $h=-k+y$, or $y=k+h$, $k \in S, h \in S_{M}$. Now

$$
\left|\phi_{\lambda}(y)-\phi_{\lambda}(0)\right| \leqq\left|\phi_{\lambda}(k+h)-\phi_{\lambda}(k)\right|+\left|\phi_{\lambda}(k)-\phi_{\lambda}(0)\right| \leqq M+M \text {. }
$$

The next step, to show that $\Gamma$ is additive, is easy:

$$
\begin{aligned}
\Gamma(h+k) & =\int_{G}(f(h+k+y)-f(y)) d \mu(y) \\
& =\int_{G}(f(h+k+y)-f(k+y)) d \mu(y)+\int_{G}(f(k+y)-f(y)) d \mu(y) \\
& =\Gamma(h)+\Gamma(k) .
\end{aligned}
$$

To complete the proof of Theorem 2.1, it remains to show that $g$ is continuous. That $\Delta_{h} g$ is continuous for each $h$ in $G$ follows from (2.3), the continuity of $\Delta_{h} f$, and the additivity of $\Gamma: \Delta_{h} g(x)=\Delta_{h} f(x)-\Gamma(x+h)+\Gamma(x)$ $=\Delta_{h} f(x)-\Gamma(h)$. Also, (2.1) and Lemma 2.1 show that $g$ is bounded. The continuity of $g$ itself is then a consequence of Lemma 2.2 .

LEMмA 2.2. Let $g$ be a function defined on an open subset $A$ of an arbitrary topological group $G$, and let $\Delta_{h} g$ be continuous on $A \cap(A-h)$ for all $h$ in some open neighborhood of 0 in $G$. If there exists a point $x_{0} \in A$ at which $g$ is discontinuous, then $g$ is unbounded on every neighborhood of $x_{0}$.

Proof. Let $g=g_{1}+i g_{2}$, where $g_{1}$ and $g_{2}$ are real-valued functions on $A$. If $g$ is discontinuous at $x_{0} \in A$, then, say,

$$
\limsup _{t \rightarrow 0} g_{1}\left(t+x_{0}\right)>g_{1}\left(x_{0}\right)+\epsilon
$$

for some $\epsilon>0$. For all $y$ in some sufficiently small open neighborhood $U$ of $x_{0}$, it follows from the continuity of $\Delta_{-x_{0}+y} g_{1}$ that

$$
\limsup _{t \rightarrow 0} g_{1}(t+y)-g_{1}(y)=\limsup _{t \rightarrow 0} g_{1}\left(t+x_{0}\right)-g_{1}\left(x_{0}\right) .
$$

Let $U^{\prime} \subset U$ be an open neighborhood of $x_{0}$. From (2.4), there exists a point $y_{1} \in U^{\prime}$ for which $g_{1}\left(y_{1}\right)>g_{1}\left(x_{0}\right)+\epsilon$. Suppose that there is a point $y_{n} \in U^{\prime}$ such that $g_{1}\left(y_{n}\right)>g_{1}\left(x_{0}\right)+n \epsilon$. Then (2.5) (with $y=y_{n}$ ) and (2.4) show that there is a point $y_{n+1} \in U^{\prime}$ such that $g_{1}\left(y_{n+1}\right)>g_{1}\left(x_{0}\right)+(n+1) \epsilon$. Hence, $g_{1}$ is unbounded above on $U^{\prime}$, thus on every neighborhood of $x_{0}$.

The proof of Theorem 2.2 would be simplified, and the assumption of second countability of $G$ eliminated, if the following result could be proved: 
Let $G$ be compact, and let $\Delta_{h} g$ be Riemann integrable on $G$ for each $h$. If $g$ is discontinuous on a set of positive measure, then $g$ is unbounded.

In the absence of such a proof, the assumption of the second countability of $G$ will be retained, so that $G$ is a compact metrizable space. Then the Riemann integrability of $g$ follows from taking $\phi(x, y)=f(x)+f(y)-f(x+y)$, $X=Y=G$ in Lemma 2.3, and the proof of Theorem 2.2 is complete.

LEMMA 2.3. Let $X$ be a compact metric space with a fixed finite regular Borel measure $\mu$, and let $Y$ be a finite measure space with measure $\nu$. Let $\phi(x, y)$ be a bounded function on $X \times Y$ which, for each fixed $x$, is a measurable function of $y$ and which, for each fixed $y$, is a Riemann integrable function of $x$. Then the function $\mathrm{g}$ :

$$
g(x)=\int_{Y} \phi(x, y) d \nu(y)
$$

is Riemann integrable on $X$.

Proof. Consider a sequence of partitions

$$
\pi_{n}: X=A_{n_{1}} \cup \ldots \cup A_{n_{m}}
$$

of $X$ into disjoint measurable subsets such that $\pi_{n+1}$ is a refinement of $\pi_{n}$ and the largest diameter of the $A_{n_{i}}$ tends to zero as $n \rightarrow \infty$. Let $\xi_{n_{i}} \in A_{n_{i}}$, and define a sequence of functionals

$$
S^{(n)} h=\sum_{i=1}^{m} \mu\left(A_{n_{i}}\right) h\left(\xi_{n_{i}}\right) .
$$

It is known [1, pp. 201, 202] that $h$ is Riemann integrable [ $\mu$ ] if and only if $\lim S^{(n)} h=S^{\infty} h$ exists for every such sequence of partitions. Let

$$
g_{n}(y)=S^{(n)} \phi(x, y)=\sum_{i=1}^{m} \mu\left(A_{n_{i}}\right) \phi\left(\xi_{n_{i}}, y\right),
$$

so that

$$
S^{(n)} g=\int_{Y} g_{n}(y) d \nu(y) .
$$

Clearly, $\left\{g_{n}\right\}$ is a sequence of measurable functions on $Y$ for which $\lim g_{n}$ exists and is given by $\lim g_{n}(y)=S^{\infty} \phi(x, y)$. Since $\mu(X)$ is finite and $\phi(x, y)$ is bounded, (2.7) shows that the functions $g_{n}$ are uniformly bounded. Hence, it follows from (2.8) and the Lebesgue Bounded Convergence Theorem that $S^{\infty} \mathrm{g}$ exists.

3. The abelian case. $(G,+)$ is henceforth assumed to be abelian and locally compact.

DEFinition. The local condition of continuity [Riemann integrability] is said to have the difference property on $G$ if the following is true for every open 
subset $A$ of $G$ : Let $f$ be defined on $A$ and have the property that, for each $h \in G, \Delta_{h} f$ is continuous [Riemann integrable] on $A \cap(A-h)$. Then there exists an additive function $\Gamma$ on $G$ such that $f-\Gamma$ is continuous [Riemann integrable] on $A$.

It is not true, in general, that $f$ itself must be continuous or Riemann integrable. Any nonmeasurable additive function on the real line provides a counterexample. There exist nonmeasurable additive functions on other groups also, as the following discussion shows.

A subset $B$ of an abelian group $G$ is a Hamel basis for $G$ if no finite subset $\left\{b_{0}, \cdots, b_{n}\right\}$ of $B$ satisfies an equation

$$
N b_{0}=r_{1} b_{1}+\cdots+r_{n} b_{n}
$$

with $N, r_{1}, \cdots, r_{n}$ integers, $N \neq 0$, and if $B$ is maximal with respect to this property. The values of an additive function $\Gamma$ on $G$ are determined by its values on $B$, for if $x \in G$, then there exist integers $N \neq 0, r_{1}, \cdots, r_{n}$ and elements $b_{1}, \cdots, b_{n}$ in $B$ such that $N x=r_{1} b_{1}+\cdots+r_{n} b_{n}$. Hence

$$
\Gamma(x)=(1 / N) \Gamma(N x)=\sum_{i=1}^{n}\left(r_{i} / N\right) \Gamma\left(b_{i}\right) .
$$

It follows from the Hausdorff Maximal Principle that the Hamel basis of any subgroup $H$ of $G$, in particular, any set consisting of a single element of infinite order, can be extended to a Hamel basis for $G$. Thus, if $\Gamma^{\prime}$ is an additive function defined on $H$, then there is an additive function $\Gamma$ on $G$ such that $\Gamma(x)=\Gamma^{\prime}(x)$ for all $x \in H$.

It is well known [8] that every locally compact abelian group $G$ is of the form $R^{n}+G_{1}$, where $R^{n}$ is Euclidean $n$-space and $G_{1}$ contains a compact open subgroup $H$. A necessary and sufficient condition in order that every additive function on $G$ be continuous is: $n=0$ and every compact subgroup of $G$ is a direct sum of cyclic groups. For if $n \geqq 1$, then any nonmeasurable additive function on $R^{n}$ can be extended as an additive function to all of $G$. If $n=0$ and $S$ is a compact subgroup of $G$, then there will be a discontinuous additive function on $G$ as soon as $S$ contains an element of infinite order. Hence, every additive function on $G$ is continuous only if

$$
S=\bigcup_{k=1}^{\infty} S_{k}
$$

where $S_{k}$ is the closed subgroup consisting of all elements of order at most $k$. $S$ being a second category space [5], there is an $S_{K}$ with nonempty interior in $S$. A finite system $\left\{s_{i}+S_{K}:(i=1, \cdots, m), s_{i} \in S\right\}$ will cover $S$, so that every element of $S$ is of order at most $K+M$, where $M$ is the maximum order of the elements $s_{i}$. Hence [4], $S$ is the direct sum of cyclic groups. Conversely, if $n=0$ and the compact open subgroup $H$ is a torsion group, then every additive function is constant on cosets of $H$, and is therefore continuous. 
THEOREM 3.1. The local condition of continuity has the difference property on $G$.

THEOREM 3.2. If $G$ is second countable, then the local condition of Riemann integrability has the difference property on $G$.

Proof of Theorem 3.1 [and 3.2]. Let $A$ be an open subset of $G$, and let $f$ be a function on $A$ such that, for each $h \in G, \Delta_{h} f$ is continuous [Riemann integrable ] on $A \cap(A-h)$. It may be assumed that $0 \in A$. Let $U$ be an open neighborhood of 0 such that $\bar{U}$ is compact and $2 \bar{U} \subset A, \bar{U}$ denoting the closure of $U$; let $G^{\prime}$ be the group generated by $U$. Then there exists [7] a compact group $H \subset U$ such that $G^{\prime} / H$ is an elementary group, that is, the direct sum of $R^{p}, q$ copies of the reals (mod 1$), r$ copies of the free cyclic group, and a finite abelian group ( $p, q, r$ non-negative integers).

The fact that $H$ is compact makes it possible to produce functions using equations like (2.1) and (2.2), so that the techniques of the previous section may be used.

Let $\nu$ be the Haar measure on $H$ for which $\nu(H)=1$. For $(x, y) \in \bar{U} \times H$, let

$$
\begin{aligned}
& g_{1}(x)=\int_{H}(f(x)+f(y)-f(x+y)) d \nu(y), \\
& f_{1}(x)=\int_{B}(f(x+y)-f(y)) d \nu(y),
\end{aligned}
$$

so that

$$
f(x)=g_{1}(x)+f_{1}(x) .
$$

Lemma 2.1, with $\phi_{x}(y)=f(x)+f(y)-f(x+y), X=\bar{U}, Y=H$, shows that $g_{1}(x)$ and $f_{1}(x)$ exist and that $g_{1}(x)$ is bounded. That the continuity of $g_{1}$ follows from that of each $\Delta_{h} f(h \in U)$ may be seen as follows: Let $h \in U$ be arbitrary, and consider points $x, x_{0}$ in $U \cap(U-h)$. Then

$$
\begin{aligned}
\Delta_{h} g_{1}(x)-\Delta_{h} g_{1}\left(x_{0}\right)= & \Delta_{h} f(x)-\Delta_{h} f\left(x_{0}\right) \\
& -\int_{H}\left(\Delta_{h} f(x+y)-\Delta_{h} f\left(x_{0}+y\right)\right) d \nu(y) .
\end{aligned}
$$

Since $\Delta_{h} f$ is uniformly continuous on $2 U \cap(2 U-h)$, it follows from (3.4) that

$$
\lim _{x \rightarrow x_{0}}\left|\Delta_{h} g_{1}(x)-\Delta_{h} g_{1}\left(x_{0}\right)\right|=0 .
$$

Thus $\Delta_{h} g_{1}$ is continuous on $U \cap(U-h)$ for all $h \in U$, and $g_{1}(x)$ is bounded on $U$, so that, by Lemma 2.2, $g_{1}$ is continuous on $U$. [That the Riemann integrability of $g_{1}$ follows from that of the $\Delta_{h} f$ is a consequence of Lemma 2.3, with $\phi(x, y)=f(x)+f(y)-f(x+y), X=\bar{U}, Y=H$.]

Next, it may be noted that while $f_{1}$ is not necessarily additive on $\bar{U}$, its 
restriction to $H$ is additive. Let $\Gamma_{1}$ be an additive function on $G$, obtained by extending the additive function on $H$ given by

$$
\Gamma_{1}(h)=\int_{H} \Delta_{h} f(y) d \nu(y) \quad(h \in H) .
$$

Then for $h \in H, x \in U$,

$$
\Delta_{h}\left(f_{1}-\Gamma_{1}\right)(x)=\int_{H}\left(\Delta_{x} f(y+h)-\Delta_{x} f(y)\right) d v(y)=0,
$$

so that $f_{1}-\Gamma_{1}$ can be considered as a function $f_{2}$ on the open neighborhood $U / H$ of the elementary group $G^{\prime} / H$. Moreover, for $h \in U, x \in U \cap(U-h)$,

$$
\Delta_{h}\left(f_{1}-\Gamma_{1}\right)(x)=\Delta_{h} f(x)-\Delta_{h} g_{1}(x)-\Gamma_{1}(h),
$$

so that, for $h^{\prime} \in U / H, \Delta_{h^{\prime}} f_{2}$ is continuous [Riemann integrable] on $(U / H)$ $\cap\left(U / H-h^{\prime}\right)$.

To conclude the proof, use is made of the fact, proved in [6] [proved in Lemma 3.1 below], that the local condition of continuity [Riemann integrability] has the difference property on $R^{p}$, hence, on every elementary group. Thus there exists an additive function $\Gamma_{2}^{\prime}$ on $G^{\prime} / H$ such that $f_{2}-\Gamma_{2}^{\prime}$ $=g_{2}^{\prime}$ is continuous [Riemann integrable] on $U / H$. Let $\Gamma_{2}^{\prime}$ be extended to all of $G$ as an additive function $\Gamma_{2}$, constant on cosets of $H$; let $g_{2}^{\prime}$ be considered as a continuous [Riemann integrable] function $g_{2}$ on $U$, constant on cosets of $H$. Thus,

$$
f(x)=g_{1}(x)+g_{2}(x)+\Gamma_{1}(x)+\Gamma_{2}(x)=g(x)+\Gamma(x) \quad(x \in U),
$$

where $g$ is continuous [Riemann integrable] on $U$, and $\Gamma$ is additive on $G$. Hence, $g$ is continuous [Riemann integrable] on all of $A$.

REMark. Continuity need not have the difference property on a group which is not locally compact. Let $G$ denote the additive group of rationals with the usual topology, and consider the function $f$ on $G$ given by

$$
f(x)=\sum_{n=1}^{\infty} n \sin (n ! \pi x) .
$$

For each rational $h, f(x+h)-f(x)$ is a finite trigonometric sum. Since the only additive functions on $G$ are continuous linear functions, it suffices to show that $f$ itself is not continuous. This may be seen from

$$
\limsup _{k \rightarrow \infty} f(1 / k !) \geqq \lim _{k \rightarrow \infty} k \sin (\pi(k-1) / k)=\pi>f(0)=0 .
$$

Lemma 3.1. Let $A$ be an open subset of $R^{p}$, and let $f$ be a function on $A$ such that, for all $h$ in some sufficiently small neighborhood of 0 in $R^{p}$, the function $\Delta_{h} f$ is Riemann integrable on $A \cap(A-h)$. Then for each connected open subset $A_{1}$ of 
$A$, there exists an additive function $\Gamma_{1}$ on $G$ such that $f-\Gamma_{1}$ is Riemann integrable on $A_{1}$.

REMARK. It is an easy consequence of this lemma that the local condition of Riemann integrability has the difference property on every elementary group.

Proof. It may be assumed that $A_{1}$ contains $3 K^{p}$ where

$$
K^{p}=\left\{\left(x_{1}, \cdots, x_{p}\right): 0 \leqq x_{i} \leqq 1, i=1, \cdots, p\right\},
$$

and that $\Delta_{h} f$ is Riemann integrable on $A_{1} \cap\left(A_{1}-h\right)$ for all $h \in K^{p}$, for otherwise a function $f^{\prime}$ could be considered: $f^{\prime}(x)=f\left(\left(x^{\prime}+x\right) / n\right), x \in 3 K^{p}, x^{\prime}$ a fixed point of $A_{1}$, and $n$ a sufficiently large integer.

We assert that it suffices to prove the existence of a point $x_{0} \in A_{1}$ and a function $F$ on $x_{0}+K^{p}$ such that: (1) $x_{0}+K^{p} C A_{1}$, (2) $F$ is Riemann integrable on $x_{0}+K^{p}$, and (3) the function $f^{*}=f-F$ satisfies $f^{*}(x)=f^{*}\left(x^{\prime}\right)$ whenever $x$ and $x^{\prime}$ are two points in (the boundary of) $x_{0}+K^{p}$ such that $x-x^{\prime} \in C^{p}$, the direct sum of $p$ copies of the integers. For then $f^{*}$ can be extended to all of $R^{p}$ by $f^{*}(x)=f^{*}\left(x^{\prime}\right)$ whenever $x-x^{\prime} \in C^{p}$. In this way, $f^{*}$ may be considered as a function on the compact group $R^{p} / C^{p}=H$. It is not difficult to see that for each $h \in H, \Delta_{h} f^{*}$ is bounded and continuous almost everywhere. From Theorem 2.2, there exists an additive function $\Gamma_{1}$ on $H$ such that $f^{*}-\Gamma_{1}=g$ is Riemann integrable on $H$. Considering $g$ and $\Gamma_{1}$ as functions on $R^{p}$, constant on cosets of $C^{p}$, we have that $f(x)-\Gamma_{1}(x)=F(x)+g(x)$ for each $x$ in $x_{0}+K^{p}$; since $\Delta_{h} f$ is Riemann integrable on $A_{1} \cap\left(A_{1}-h\right)$ for each $h \in K^{p}$, and since $A_{1}$ is connected, it follows that $f-\Gamma_{1}$ is Riemann integrable throughout $A_{1}$.

It remains to prove the existence of a pair $x_{0}, F$ having the required properties. In fact, for all $x_{0} \in K^{p}$ outside some set of measure 0 , the following function has these properties:

$$
\begin{aligned}
F(x)=\left.\sum(-1)^{k+1} x_{i_{1}} \cdots x_{i_{k}} \Delta_{i_{i_{1}}} \cdots \Delta_{e_{i_{k}}} f(x)\right|_{x_{i_{j}}-x_{i j}{ }^{0}} \\
\left(j=1, \cdots, k ; x \in x_{0}+K^{p}\right),
\end{aligned}
$$

where the summation is taken over all nonempty subsets $\left(i_{1}, \cdots, i_{k}\right)$ of $(1, \cdots, p)$. Here, $\epsilon_{j} \in R^{p}$ has $\delta_{h}^{j}$ as its $h$ th coordinate; further, $x_{h}^{0}$ denotes the $h$ th coordinate of $x_{0}$. It is easily seen that

$$
\Delta_{\epsilon_{h}}[f(x)-F(x)]_{x_{h}=x_{h}}=0
$$

(hint: consider pairs of subsets $S, S^{\prime}$ of $(1, \cdots, p)$ with $S=S^{\prime} \cup\{h\}$ ), hence, $f^{*}=f-F$ has the required "periodicity," when $x \in x_{0}+K^{p}$. Further, $\Delta_{h} f$ is defined and bounded on $2 K^{p}$ for each $h \in K^{p}$, hence $F(x)$ is bounded on $K^{p}$ for each $x_{0} \in K^{p}$. Suppose that $F$ is not continuous almost everywhere in $x_{0}+K^{p}$. Then for at least one nonempty subset $\left(i_{1}, \cdots, i_{k}\right)$ of $(1, \cdots, p)$, the function

$$
\left.G\left(x_{1}, \cdots, x_{p}\right)\right|_{x_{i_{1}=x_{i_{1}}{ }^{0}, \cdots, x_{i_{k}}=x_{i_{k}}{ }^{0}}}
$$


would not be continuous almost everywhere on $x_{0}+K^{p}$, where

$$
G(x)=\Delta_{\epsilon_{i_{1}}} \cdots \Delta_{\epsilon_{i_{k}}} f(x) .
$$

But $G(x)$ is itself continuous almost everywhere on $2 K^{p}$, thus, the latter event can happen only on a set of points $x_{0}$ of measure 0 .

\section{REFERENCES}

1. N. Bourbaki, Intégration, Actualités Sci. Ind. No. 1175, Hermann, Paris, 1952.

2. N. G. de Bruijn, Functions whose differences belong to a given class, Nieuw Arch. Wisk. (2) 23 (1951), 194-218.

3. - A difference property for Riemann integrable functions and for some similar classes of functions, Nederl. Akad. Wetensch. Proc. Ser. A 55 (1952), 145-151.

4. I. Kaplansky, Infinite Abelian groups, Univ. of Michigan Press, Ann Arbor, Mich., 1954 ; p. 17.

5. J. L. Kelley, General topology, Van Nostrand, New York, 1955, p. 200. 28-56.

6. J. H. B. Kemperman, $A$ general functional equation, Trans. Amer. Math. Soc. 86 (1957),

7. L. Pontrjagin, Topologische Gruppen, Vol. 2, B. G. Teubner, Leipzig, 1958, pp. 54-57.

8. A. Weil, L'intégration dans les groupes topologiques et ses applications, Actualités Sci. Ind. No. 869-1145, Hermann, Paris, 1953, p. 110.

UNIVERSITY OF WISCONSIN, MiL WAUKEe, Wisconsin 